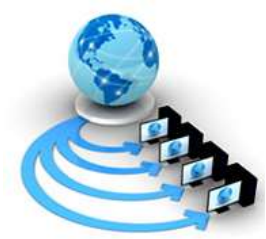

Volume 9, No. 6, November-December 2018

International Journal of Advanced Research in Computer Science

RESEARCH PAPER

Available Online at www.ijarcs.info

\title{
ATTENDANCE USING FACE RECOGNITION
}

\author{
Sai Kiran Chintalapudi \\ School of Computer Science and Engineering \\ Vellore Institute of Technology \\ Vellore, India
}

\author{
Prajwal Reddy \\ School of Engineering for Computers \\ Sreenidhi Institute of Science and Technology \\ Hyderabad, India
}

\author{
Keerthi Shrikar Tatapudi \\ School of Computer Science and Engineering \\ Vellore Institute of Technology \\ Vellore, India
}

\begin{abstract}
Professors who teach at Universities spend a lot of time for taking attendances in different classes (slots). If theclassstrengthexceeds60,thenit will be a problem for them as they should take the attendance and teach also in the same hour. If they should mark present to a student later, then they must search for his name or any of his unique ID. Also, students will try to fake the attendance as they have to just say yes for a number which will increase the amount of proxies in a class. As it doesn't matter for teachers, they somehow are satisfied until any one is caught red-handed. Here, we have put to use the machine learning algorithms to register the attendance using face recognition.
\end{abstract}

Keywords: Attendance, recognition, product, histogram, functions.

\section{INTRODUCTION}

The purpose of this project paper is to explain in detail about the requirements of our project. This is intended to the users, and is to be used to know about the project and verify the correct running of system. It will explain the purpose and features of the system, the interfaces of the system, what the system will do, the constraints that it must operate. In addition, users can use product functions and use case diagrams to learn how the product can be used.

\section{PRODUCT SCOPE:}

Attendance using face recognition system is a software application which automatically updates the attendance in classrooms. As every faculty has a habit of bringing their laptops to their respective classes, the attendance of the students of that class will be taken by using the camera present to the laptop or by using an external camera piece. By using this, lecturers can maintain their valuable time in a perfect way. All the faculty should do is entering the details of students of a class in separate folder, so that this softwarewillsearchforthestudent'sfacewhenheappearsforthes canandwillmarkhim present if his ther photo is present in the folder. This software is strictly for the lecturers but not for the students as they might mark up their attendance and be benefitted without attending to class.

\section{LITERATURE SURVEY}

\section{EXISTING SYSTEM}

Pedestrian detection is the existing project which is widely used all over the world. It is used in a larger basis. It is much more useful thing for the detection of people for catching criminals and to detect the robberies.

\section{APPLICATIONS OF ATTENDANCE USING FACE}

\section{RECOGNITION}

1. To remove the proxies in the attendance.

2. Creating genuine records in all the colleges.

3. Decrease the time taken for attendance by the lectures.

4. To decrease the work load of every faculty, so that they can concentrate on more about educating people.

5. As the world is updating in a faster pace, it is much needed for every institution to stay updated and make everything mechanicalized.

6. As it is an offline based software, it will never depend on the network problem or the lack of internet.

7. Lesser paper work.

\section{RISKS IN IMPLEMENTATION OF ATTENDANCE USING FACE RECOGNITION}

1) The faculty must enter the details of all the students in a folder, a single miss match will lead to some more errors during attendance.

2) Requires high clarity camera for detection of face to make the face look more genuine.

If more number of student's data is present in the folder, it requires more time for the software to scan and detect faces and mark presentlabsent. 


\section{METHODOLOGY}

Rule based approach is used by defining various rules for getting the opinion, created by tokenizing each sentence in every document and then testing each token, or word, for its presence. If the word is there and has with a positive sentiment, a +1 rating will be applied to it. Each post starts with a neutral score of zero, and is considered positive, if the final polarity score is greater than zero, or negative if the overall score is less than zero. After the output of rule based approach it will check or ask whether the output is correct or not. If the input sentence contains any word which is not present in the database which may help in the analysis of movie review, then such words are to be added to the database. This is supervised learning in which the system is trained to learn if any new input is given.

To implement this project, three phases are to be executed:

\section{DETECTION}

First phase is detection. This is very important phase because student need to be very attentive in expressing different facial expressions. He need to concentrate the camera. If any mistake happens, attendance will be marked too, due to their similar faces. Here there is one more task, Student need to enter the registered no carefully. This phase captures 25 photographs of student.

It will be saved in a folder called "Images". These photos are the base for the next processes/phases.

\section{TRAINING THE CODE}

The second phase is Training the code. This section is where actual process happens behind the screens. All the photos with the same name, are converted in to a huge matrix and that will be saved in to a "meta language".

Code uses these number in matrix to recognize the face of a particular

student.Onanobservation, eachstudentgetsa4mbofdata. So, imagine how big the matrix would be. We can view it using Notepad++ like editors. '.yml' will be stored in a separate folder called 'Trainer'.

\section{RECOGNITION}

The third and final phase is recognizing the faces. Our program uses LBPH (Local Binary Pattern Histogram) face recognizer to recognize the faces. Data to that recognizer is given by yml file. Using that data it recognizes the Face. We used file operation to mark attendance. Automatically, as soon as it recognizes, Attendance will be marked.

\section{IMPLEMENTATION: -}

In Supervised Machine learning techniques, two types of data sets are required: training data set and test data set. A model is created based on the training data set which is employed over the new/unseen text for classification purpose.

An automatic classifier learns the classification factors of the document from the training set and the accuracy in classification can be evaluated using the test set. Various machine learning algorithms are available that can be used very well to classify the documents.

\section{COMPARATIVE ANALYSIS}

Time can be saved as all the students need to do is to stand before the camera for a fraction of seconds and his attendance will be marked present. If his details are not present, they the lecturer had either missed to enter his data or the student is not from this class. This will reduce the time of taking attendance and will also increase the genuine records in the basis of attendance.

\section{TESTING REPORT}

1. While entering the registration number, the student should just enter the integer part leaving the characters' part.

2. The student must stand for about 20 seconds to scan his face with different expressions, so that in future, no matter what expression helshe gives the software will be able to recognize him.

3. The user (faculty) must have the Microsoft Excel preinstalled in the system.

4. The teacher must take responsibility to enter the details of the students in different folders.

\section{RESULTS AND FUTURE SCOPE}

We have overcome the problems faced by the faculties during their classes. This software doesn't even require much space in the hard disk. It can be used by all the faculties whoever has laptops. It is not a complicated task as it is developed with a decent UI so that beginners can also use it with ease. Though we faced several problems while working for this software, we had overcome through all of them and brought this product within the required timeline. In the later updates, we will try to implement more new features like walk a way feature, the student just needs to walk his id card will be scanned at the door-step and will mark his attendance in the laptop by sending the data from a sensor which is established at the doors of the classrooms. 


\section{SCREENSHOTS OF SOFTWARE}
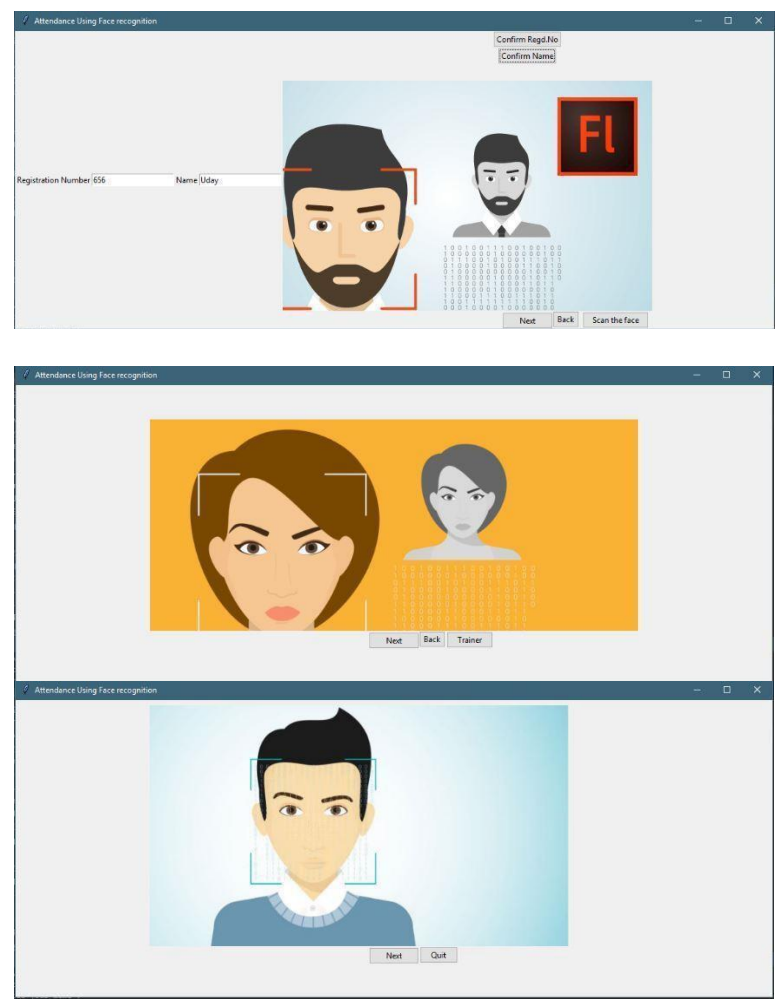

\section{REFERENCES}

1. Automatic Attendance Management System Using Face Recognition Jomon Joseph1, K. P.Zacharia2.
2. M. Hu and B. Liu, "Mining and summarizing customer reviews," Proceedings of the tenth ACM international conference on Knowledge discovery and data mining, Seattle,2004, pp. 168-177.

3. B. Pang, L. Lee, and S. Vaithyanathan, "Thumbs up?: sentiment classification using machine learning techniques," Proceedings of the ACL-02 conference on Empirical methods in natural language processing, vol.10, 2002, pp. 79-86.

4. K. Dave, S. Lawrence, and D. M. Pennock, "Mining the peanut gallery: Opinion extraction and semantic classification of product reviews," Proceedings of WWW, 2003, pp. 519-528.

5. R. Prabowo and M. Thelwall, "Sentiment analysis: A combined approach" , Journal of Informetrics, vol. 3, pp.143-157, 2009.

6. Ankitha Srivastava, Dr.M.P. Singh, "Supervised SA of product reviews usin Weighted k-NN Algorithm," 2014 11th International Conference on Information Technology.

7. Ji Fang and $\mathrm{Bi}$ Chen, "Incorporating Lexicon Knowledge into SVM Learning to Improve Sentiment Classication", In Proceedings of the Workshop on Sentiment Analysis where AI meets Psychology (SAAIP), pages 94-100, 2011.

8. A. Mudinas, D. Zhang, M. Levene, "Combining lexicon and learning based approaches for conceptlevel sentiment analysis", Proceedings of the First International Workshop on Issues of Sentiment Discovery and Opinion Mining, ACM, New York,NY, USA, Article 5, pp. 1-8, 2012. 